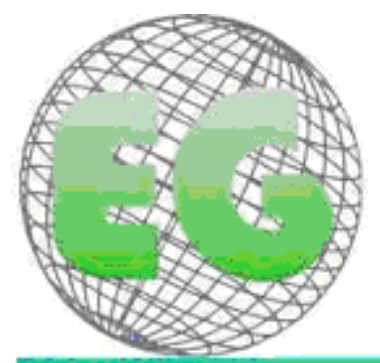

15SN 1696-6*5:

\title{
LAS ESPECIALIDADES Y LOS VÍNCULOS CON LA FORMACIÓN CONTINUA DEL ENFERMERO: REPERCUSIONES PARA LA ACTUACIÓN EN EL MUNICIPIO DE RIO DE JANEIRO
}

AS ESPECIALIDADES E OS NEXOS COM A FORMACÃO CONTINUA DO ENFERMEIRO: REPERCUSSÕES PARA A ATUAÇÃO NO MUUICIPIO DO RIO DE JANEIRO

* Cavalcanti Valente, GS., "*Viana, L de O., ***Garcia Neves, I.

*Doctora em Enfermería. Profesora Adjunta del Departamento de Fundamentos de Enfermería y Administración de la Universidad Federal Fluminense - UFF ** Profesora Titular del Departamento de Metodologia de Enfermería de la Escuela de Enfermería Anna Nery ${ }^{* * *}$ Graduada em Enfermería. Brasil.

Palabras clave: Formación continua., Especialidades. Enfermería.

Palavras chave: Formação contínua, Especialidades, Enfermagem.

\section{RESUMEN}

Este estudio objetivó captar cómo se está construyendo el proceso de formación continua de la enfermera a través de las especialidades, así como las repercusiones que la especialización trae para la formación y el funcionamiento de la enfermera en la asistencia de Enfermería. Trabajamos con el material bibliográfico resultante de la investigación científica publicada en los últimos diez años, siendo utilizado el análisis del contenido para el tratamiento de los datos encontrados. Destacó como dato relevante que la especialización compone un instrumento necesario en la la formación continua de la enfermera para que desarrolle las competencias para actuar en un mercado de trabajo cada vez más exigente y competitivo.

\section{RESUMO}

Este estudo objetivou captar como está sendo construído o processo de formação continua do enfermeiro através das especialidades, bem como as repercussões que a especialização traz para a formação e atuação do enfermeiro na assistência de Enfermagem. Trabalhamos com material bibliográfico resultante de pesquisas cientificas de Enfermagem publicadas nos 
últimos dez anos, sendo utilizada a análise de conteúdo para tratamento dos dados encontrados. Destacou-se como dado resultante que a especialização compõe um instrumento necessário à formação continua do enfermeiro para que desenvolva as competências para atuar num mercado de trabalho cada vez mais exigente e competitivo.

\section{INTRODUCCIÓN}

En la actualidad, la introducción de nuevas tecnologías en el mercado del trabajo de la salud ha impuesto algunos cambios en la fuerza del trabajo del sector, donde hay una necesidad de contrato de profesionales cUalificados, que estén capacitados para utilizar las innovaciones en el diagnóstico y terapéutica. Ampliamos este fenómeno en el campo de la enfermería, puesto que un número cada vez mayor de enfermeras ha estado persiguiendo la mejora continua a través de cursos de postgrado.

La especialización en el cuidado viene prácticamente convirtiéndose en un requisito para la complementación y la sedimentación del proceso de aprendizaje conseguido en el curso de pregrado, proporcionando las herramientas necesarias para la práctica profesional. Ya no es posible permanecer solamente con el conocimiento adquirido en el pregrado. Esto pone la enseñanza de postgrado (en sentido amplio) como una de las posibilidades que tenemos actualmente para calificar las enfermeras para la práctica profesional y, por tanto, contribuir a la expansión y transformación de la enfermería brasileña.

En el contexto del mundo globalizado, nos encontramos ante la necesidad de la formación continua y perfeccionamiento de los recursos humanos para las actividades sanitarias. La enfermera tiene que hacer frente a problemas relacionados con la salud de los clientes, sin prejuicio de la calidad. Todo este problema se puede apoyar en afirmaciones de los autores, cuando señalan que con el aumento de la vida esperan, por lo tanto, el aumento de la esperanza de la vida productiva, hacen frente cada vez más a cambios de paradigmas, de procesos tecnológicos y de la gerencia del trabajo. ${ }^{(1)}$

El postgrado se convierte así, en la más duradera y diversificada estrategia de la educación continua. Además, el enfoque intersectorial e interdisciplinario será de renovar y crear espacios para la acción, como nunca se vio en la historia de la humanidad, por lo tanto es necesario buscar, en cada momento, en la universidad, el conocimiento no agregado en la formación básica.

La especialización es un proceso de la educación que objetiva ampliar los conocimientos y las concepciones del área, la preparación de profesionales para intervenir y transformar la práctica. El especialista se dedica a una rama de su profesión, de una especialidad profesional de acuerdo con su capacidad o interés. La elección de una especialidad profesional se caracteriza por ser un momento importante de la transformación personal y social, cargada de simbolismo y significaciones individuales y colectivas, no constituyendo, por lo tanto, una opción simple y fácil como puede parecer ${ }^{(1)}$.

En la salud, según los autores anteriores, las profesiones también caminan, día a día, a la especialización de los conocimientos y del cuidado, a medida que los nuevos conceptos y tecnologías se desarrollan, surgiendo como consecuencia, nuevos campos de actividad e investigación. En el contexto de las profesiones de la salud, la 
enfermería ocupa una posición impar en las instituciones, pue es numéricamente significativa, casi exclusivamente femenina, desarrollando las actividades centradas en el cuidado en la salud de la gente.

En los años 70, hubo un incremento científico indiscutible que llevó a un gran avance en materia de salud que exigió de los profesionales una necesidad intensa de estudiar, en la búsqueda para seguir tal crecimiento. (2) Por otra parte, la especialización en enfermería es un tema controvertido para la categoría, especialmente en lo que respecta a la absorción de las enfermeras por el mercado, ya que los puestos de trabajo rara vez se ofrecen conforme la experiencia específica realizada.

Hay una contradicción evidente en enfermería: rara vez surgen puestos de trabajo de una especialización. Por lo tanto, las enfermeras tienden a insertarse al azar en el mercado laboral, basado solamente en la demanda ofrecida. ${ }^{(3)}$

\section{OBJETO DE ESTUDIO}

\section{La enseñanza de postgrado, como un factor de apoyo para la formación continua de la enfermera.}

Por medio de la problemática presentada, los objetivos son: Presentar una visión general de la especialización en enfermería en Río de Janeiro - Brasil; identificar el impacto provocado por la formación para la práctica profesional de la enfermera.

Siempre hemos estado interesados en cuestiones relativas a la formación de los profesionales de enfermería en marcha en el mercado laboral, lo que nos lleva a prestar atención al hecho de que el programa de pregrado ofrece una visión integral de la enfermería, y la postgraduación es quien aporta la visión de un especialista en enfermería, para hacer frente a la necesidad de profundizar y actualizar en lo relativo al logro de la autonomía profesional, y para una mejor inserción en el mercado laboral, muy sofisticado y tecnológico. (4)

En este enfoque, consideramos que la especialización es la etapa de la formación que irá a contribuir al aprender, y así estimular la capacidad de entender cómo el conocimiento se produce en varias áreas, creando condiciones para una educación permanente, que lleve a la enfermera a estar cada vez más comprometida con la sociedad y sus problemas de salud, haciéndose en competente frente a los retos del siglo $\mathrm{XXI}$, construyendo una conciencia crítica con respecto al contexto en el cual está inserto.

\section{MÉTODO}

Este estudio sigue la línea de la investigación y el área temática de Educación en Enfermería, en forma bibliográfica, lo que se puede definir como la investigación sobre los documentos y fuentes secundarias, y abarca toda la literatura ya publicada en relación con el objeto de estudio debido a las publicaciones, boletines, periódicos, revistas, libros, investigaciones, monografías, tesis, material cartográfico, y otros. Su propósito es poner al investigador en contacto directo con todo lo que estaba escrito, dicho o filmado sobre un tema. (5) 
Buscando informaciones que ayuden a comprender el proceso de especialización de la enfermería, se realizó una encuesta de artículos científicos relacionados con ésta, producidos y publicados entre los años 2000 y 2009. Sin embargo, encontramos una corta lista de posibilidades, porque el tema todavía está sin explorar y tiene pocas publicaciones científicas recientes. Por lo tanto, los resultados no se muestran suficientes, y también buscamos una investigación sobre la producción científica de enfermería especializada, publicada en otros períodos.

Utilizamos los medios electrónicos como la Biblioteca Regional de Medicina (BIREME), en cuyas bases de datos se realizaron búsquedas: Scientific Electronic Library Online (SciELO), Base de Datos de Enfermería (BDEN) y América Latina y el Caribe en Ciencias de la Salud ( LILACS) Y también las bibliotecas de las escuelas / colegios de enfermería pública y privada en Río de Janeiro.

Como el tema está directamente relacionado con el área de la enseñanza, la investigación la llevamos a cabo en los sitios de interés como la CAPES (Coordinación de Perfeccionamiento de Nivel Superior), el MEC (Ministerio de Educación); INEP (Instituto Nacional de Estudios e Investigaciones Educacionales) (IBGE organizaciones Instituto Brasileño de Geografía y Estadística), DBO (Clasificación Brasileña de Ocupaciones) y clase (ABEN, COFEN, COREN), entre otros. Ocupados en el proceso de recopilación de datos, palabras clave como: conocimientos, experiencia, especialidad, postgrado, enfermería, enfermera.

El análisis del material se basó en el método llamado análisis de contenido, que puede definirse como un conjunto de herramientas metodológicas que se prestan al estudio de las comunicaciones. Y, por tanto, puede ser utilizado en el análisis de las comunicaciones que se producen entre el emisor y el receptor, ya sean individuos 0 grupos. (6)

Después de elegir el material, el siguiente paso fue la realización de una lectura inicial, cuando se comenzaron a imprimir las directrices para el análisis. De esta actividad, hemos hecho la estructura de índices y la elaboración de indicadores. Este fue un trabajo preparatorio para el análisis. Por último, tratamos a los resultados obtenidos y el trabajo de la inferencia y la interpretación. Así que en el análisis final, buscamos el establecimiento de un vínculo entre los datos de texto y los objetivos previstos.

\section{ENSEÑANZA DE LA ENFERMERÍA EN EL BRASIL Y LAS ESPECIALIDADES}

Hay una relación fuerte y estrecha entre la historia de la enfermería en Brasil y la transformación general de la estructura social brasileña, por lo tanto es esencial usar la ruta más larga adoptada por la enfermería en el país como fuente de datos que permitan la comprensión de las cuestiones relativas al proceso de transición experimentado por la categoría con respecto a su experiencia.

La escuela de Enfermería Anna Nery de la Universidad Federal del Rio de Janeiro, fue un foro para las importantes decisiones políticas e intelectuales durante cinco décadas, comenzando sus actividades en el año 1923. De ella salieron líderes que construyeron el terreno sólido del desarrollo de la enseñanza, de la investigación y atención de enfermería en Brasil (7). 
Entre los años 40 y 60, hubo una gran expansión numérica de los hospitales y las escuelas de enfermería en Brasil, que se tradujo en la necesidad de crear herramientas para estandarizar y estructurar los cursos de graduación en Enfermería, lo que llevó y permitió el principio de las discusiones sobre cursos de postgrado, con el nombre de especialización.

La tendencia a la formación especializada en enfermería brasileña tuvo sus comienzos en función de la demanda derivada de los problemas socioeconómicos y la salud de los sectores especialmente marginados de la población, especialmente los que viven en centros urbanos. En los años 70, después de la reforma universitaria, implementada por la Ley 5540/68, la enseñanza de enfermería en el Brasil pasa por un proceso de normalización, donde a través del Parecer no 163/72, los programas son definidos y se guía por normas comunes a todas las instituciones de educación superior. (8)

Con este dictamen, el curso de enfermería abarca en la actualidad las ciencias básicas, las disciplinas profesionales y la proporción de cualificaciones específicas, a saber: Enfermería de Salud Pública, Enfermería Obstétrica y Enfermería MédicoQuirúrgica y en la actualidad se da un paso importante en la dirección a las especializaciones en Enfermería, que cobran más ímpetu y la aceptación en el ámbito académico, dado el entendimiento de que estas habilidades sirvan como preespecializaciones.

A pesar de la existencia de los cursos de Postgrado en Enfermería en sentido estricto (máster y doctorado) desde los años 40, fue sólo después del año 1974, cuando ganaron fuerza y la atención de las enfermeras. Este cambio se debió principalmente a una mayor demanda de docentes preparados para la educación superior, que necesitaba tener un número de profesores cualificado. (8)

La especialización en el oficio de enfermera tiene relación con el contexto de la política de la salud en Brasil y con la evolución del proceso de la reforma sanitaria, desde la creación del Instituto Nacional de Asistencia Social (INPS - 1966) hasta su conclusión, con la implantación del único Sistema de Salud (SUS - 1990), que impuso demandas y ofertas específicas. Esta relación se hizo más estrecha cuando la asociamos con el hecho de la privatización del sector salud, que ofrecen la posibilidad de alianzas y la formación de los grupos para el seguro de salud, promoviendo la necesidad de oferta de profesionales en cantidad y calidad suficientes para satisfacer las necesidades del mercado de trabajo. (9)

El crecimiento de la industria farmacéutica y hospitalaria obligó a la asimilación de las tecnologías en el campo de los hospitales y dirigió a los funcionarios de salud para la manipulación y el consumo de estos materiales, creando nuevas necesidades. Así, el desarrollo de las especialidades en enfermería, se caracteriza en un contexto del crecimiento de la propia profesión, que inicialmente se centró en la salud pública, y pasa a satisfacer un creciente mercado hospitalario. (8)

Los datos sobre los cursos de postgrado, presenciales y a distancia, indican una expansión del sector en relación con el número de instituciones que ofrecen estos cursos. Del cruce de los datos se infiere que casi la mitad de las Instituciones de Educación Superior (IES) ofrecen cursos de postgrado en Brasil en sentido amplio, y que este nivel de educación se limita a las universidades y escuelas integradas. (9) 
Es importante destacar, sin embargo, que al considerar el número de matrículas en los cursos ofertados, se observa que el $50 \%$ de la matrícula total en el país en términos generales de postgrado, presencial y a distancia, se encuentra en las instituciones del sudeste brasileño. Si se comparan de las instituciones públicas y privadas, se observa que estas últimas ofertan un mayor número de cursos presenciales.

De acuerdo con la Clasificación Brasileña de Ocupaciones - CBO, las enfermeras, tienen doce clases o profesiones: enfermera, enfermera auditor, enfermera de a bordo, enfermera de sala quirúrgica, enfermera de Cuidados Intensivos (UCI enfermera), enfermera del trabajo, nefrólogo enfermera, Enfermera neonatólogo, matrona (partera), enfermera psiquiátrica, enfermera de Salud Pública, y enfermera pediátrica.

La Residencia en Enfermería, creada en Brasil en la década de 1960, pasó por momentos de grandes desafíos para consolidarse como un programa de enseñanza/aprendizaje/trabajo. En su trayectoria realizó movimientos sociales 'reivindicatorios y luchas importantes para la conquista del espacio en las instituciones de salud. Enfrentándose a desafíos y a distorsiones en lo referente a sus conceptos filosóficos y éticos, los programas en general, han buscado la manera que tengan en cuenta no sólo el uso de la fuerza laboral de las enfermeras residentes, sino la estructuración de un modelo pedagógico que cumpla los requisitos de la formación especializada de los profesionales. ${ }^{(10)}$

En esta búsqueda, los programas han evolucionado hasta desarrollar un nuevo canal teórico que apoya la demanda del conocimiento cotidiano y las estructuras de la educación, destacando los programas de investigación y extensión universitaria. En la actualidad, algunos programas han sido diseñados con la integración en las instituciones de educación superior, lo que les garantiza el título de especialización. La residencia es una especialización en Enfermería con $80 \%$ de las actividades centradas en la práctica y $20 \%$, en la teoría, con la oferta de bolsas - para ayudar a los profesionales que opten por realizar la misma. La carga de trabajo total anual varía entre 2880 y 3520 horas. (4)

La residencia en enfermería fue reconocida como modalidad de formación de postgrado, dirigida a la educación en servicio y destinada a las categorías profesionales que integran el área de la salud, según el no 11.129 de la Ley. de 30/06/2005; publicado en la Gaceta Oficial de 01//07/2005. ${ }^{(4)}$

\section{CURSOS DE ESPECIALIDAD EN ENFERMERÍA EN RIO DE JANEIRO}

Tabla 1 - Lista de los Cursos de Especialidad de Enfermería de los Colegios / Escuelas de Enfermería en Río de Janeiro - Brasil (2009-2010)

\begin{tabular}{|c|c|}
\hline INSTITUIÇÃO & CURSO DE ESPECIALIZAÇÃO \\
\hline UNIGRANRIO & $\begin{array}{c}\text { Enfermería del Trabajo, Cardiologia, salud pública, UTI, } \\
\text { neonatal, salud de la familia, centro quirúrgico, central del } \\
\text { material y esterilización, salud de la mujer }\end{array}$ \\
\hline UNIRIO & $\begin{array}{l}\text { Clínica y generalidad quirúrgica, clínica y Cardiologia } \\
\text { quirúrgico, clínica y salud quirúrgica de Ortopedia, del } \\
\text { público, clínica y quirúrgica en hematología. }\end{array}$ \\
\hline
\end{tabular}




\begin{tabular}{|c|c|}
\hline SOUZA MARQUES & $\begin{array}{c}\text { Enfermería del Trabajo, Enfermería Obstétrica, Enfermería } \\
\text { en Stomatherapy. }\end{array}$ \\
\hline SÃO CAMILO & $\begin{array}{l}\text { Salud de la Familia, Salud Pública, Salud Pública con } \\
\text { énfasis en el PSF, Oncología, Enfermería del Trabajo, } \\
\text { Centro quirúrgico, Neonatal, Nefrología, Emergencia, Home } \\
\text { Care, Auditoría en Enfermería, Enfermería del Trabajo, } \\
\text { cardiología, centro quirúrgico, terapia intensiva adultos, } \\
\text { terapia intensiva pediátrica, obstetricia, gerontología. }\end{array}$ \\
\hline UERJ & $\begin{array}{l}\text { Enfermería Obstétrica, Neonatal, Intensivista, Salud de la } \\
\text { Familia y de la Comunidad. Enfermería del Trabajo, } \\
\text { Stomatherapy, Gestión en salud de la familia }\end{array}$ \\
\hline UNESA & $\begin{array}{l}\text { Enfermería en UTI, Enfermería Neonatal e Pediátrica, } \\
\text { Enfermería de cuidados Intensivos, Enfermería del trabajo, } \\
\text { Enfermería en emeraencia. }\end{array}$ \\
\hline GAMA FILHO & $\begin{array}{c}\text { Enfermería Dermatológica, Enfermería del Trabajo, } \\
\text { Enfermería Intensiva de Alta Complejidad, Nefrologia, } \\
\text { Obstétrica, Prevención y Control de las Infecciones en el } \\
\text { Hospital, Cardiología, Cirugía, Emergencia y trauma, } \\
\text { Hemoterapia y Hematología, Salud de la mujer, } \\
\text { Neonatología, Oncología, Pediatría. }\end{array}$ \\
\hline UFRJ & $\begin{array}{c}\text { Enfermería del Trabajo, Enfermería Obstétrica, Enfermería } \\
\text { Pediátrica, Clientes de mayor complejidad, Enfermería } \\
\text { Cardiológica, Salud Pública. }\end{array}$ \\
\hline FIOCRUZ - IFF & $\begin{array}{l}\text { Enfermería Pediátrica, Enfermería Neonatal, Ética Aplicada } \\
\text { y bioética. }\end{array}$ \\
\hline INCA & Enfermería Oncológica \\
\hline
\end{tabular}

Fuente: Home pages de las Universidades. Disponible en sitio electrónico de las respectivas Facultades/Escuelas de Enfermería del Município del Rio de Janeiro. Acceso en: 02/ 2010. 


\section{LAS ESPECIALIDADES}

Tabla 2 - El Consejo Federal de Enfermería (COFEN), a través de la Resolución no 290/2004 de 24 de marzo de 2004, fija como especialidades de Enfermería, de competencia del Enfermero, las siguientes calificaciones:

\begin{tabular}{|l|l|}
\hline 1.Aeroespacial & 22. Hemodinamica \\
\hline 2. Asistencia al Adolescente & 23. Home Care \\
\hline 3. Atendimento Pré-Hospitalar & 24. Infección Hospitalar \\
\hline 4. Banco de Leite Humano & 25. Informática \\
\hline 5. Cardiovascular & 26. Nefrologia \\
\hline 6. Central de Material e Esterilización & 27. Neonatologia \\
\hline 7. Centro quirúrgico & 28. Nutrición Parenteral \\
\hline 8. Clínica quirúrgica & 29. Obstetrícia \\
\hline 9. Clínica Médica & 30. Oftalmologia \\
\hline 10. Dermatologia & 31. Oncologia \\
\hline 11. Diagnóstico por Imagen & 32. Otorrinolaringologia \\
\hline 12. Enfermedades Infecciosas & 33. Pediatria \\
\hline 13. Educación en Enfermería & 34. Perícia y Auditoria \\
\hline 14. emergencia & 35. Psiquiatria e Salud Mental \\
\hline 15. Endocrinologia & 36. Salud Colectiva \\
\hline 16. Endoscopia & 37. Salud da Familia \\
\hline 17. Estomaterapia & 38. Sexologia Humana \\
\hline 18. Ética e Bioética & 39. Trabajo \\
\hline 19.Gerenciamento Servicios de Salud & 40. Traumato-Ortopedia \\
\hline 20.Gerontologia e Geriatria & 41. Terapia intensiva \\
\hline 21.Ginecologia & 42. Terapias Naturales/Tradicionales \\
\hline
\end{tabular}

Fuente: Disponible en: <http://www.portalcofen.com.br>Acceso: 02/ 2010.

Hoy, con el creciente número de escuelas de enfermería y en consecuencia de plazas que ofrecen los cursos de la Especialización, estos cursos siguen las normas de la Resolución no 1 CNE / CES (Consejo de Educación Superior del Consejo Nacional de Educación), de 03 de abril 2001, porque ninguno de ellos presenta una carga de trabajo inferior al determinado en la resolución, cuyo mínimo es de 360 horas.

\section{PRESENTACIÓN Y ANÁLISIS DEL MATERIAL}

\section{El mercado de trabajo requiere una formación especializada}

La división del trabajo en las tareas parciales, también con experiencia en el sector de la salud, sobre todo en las últimas cuatro décadas, ha seguido la evolución de la sociedad durante este tiempo y se dirigen a responder a las demandas para una mayor productividad. Los nuevos tiempos exigen una comprensión de los cambios intensos y rápidos que ocurren en el mundo, en educación, en la vida.

La salud es un sector importante en el mercado laboral en el país. Al igual que todos los servicios para el consumo colectivo, se enlaza a un conjunto de determinantes políticos y económicos que subyacen en el mercado de trabajo en general. Se podría 
decir que el dinamismo y las características de sus empleos se vieron afectados por el crecimiento de los servicios de producción y la reforma del sector, lo que conllevó importantes transformaciones en la estructura ocupacional, de perfil cualitativo y cuantitativo de la fuerza de trabajo en salud. (9)

Naturalmente, la enseñanza en enfermería tiene que adaptarse a los cambios impuestos a fin de satisfacer principalmente las necesidades y las demandas del mercado laboral, que con el tiempo se convirtió en algo cada vez más claro y sofisticado.

La creciente automatización de los procesos productivos ha transformado el perfil de la población económicamente activa, puesto que se trata de mano de obra cada vez más especializada y preparada para hacer frente a los avances propuestos por la reingeniería. (3)

Esta realidad impone un reto para cubrir una expansión de la demanda, con competencias y conocimientos técnicos, para garantizar la asistencia que puede contribuir a la expansión de la vida y la reducción de secuelas. Una cuestión importante que merece atención es que el número de enfermeras que entraron en el mercado de trabajo semestralmente es mucho mayor que en el mismo periodo una década atrás, influyendo directamente en el mercado, que es muy selectivo. La enfermera no es inmune a todas las transformaciones que están ocurriendo en el mundo del trabajo.

La receptividad de las enfermeras a especializarse se atribuye a la constante evolución técnica y científica, que presenta demandas y reflexiones sobre la práctica profesional, lo que resulta en la necesidad de actualizar los conocimientos, del refuerzo o de la adquisición de nuevas habilidades con el fin de garantizar el desempeño profesional con calidad y seguridad. (8)

De hecho, el trabajo en los hospitales especializados, exige profesionales con la preparación compatible. Debemos reconocer que hay fuerzas convergentes que conducen a la senda de la especialización, al menos en los grandes centros urbanos.

¿Qué dirían los dirigentes y funcionarios del gobierno si no hubieran centros de excelencia como los que existen en Sao Paulo y Río de Janeiro? Estas grandes ciudades tienen necesidad del equipos especialistas, no sólo en cursos especializados sino también de maestría y doctorado. (9)

Creemos que los profesionales que trabajan en estos centros están obligados a especializarse en todas las tecnologías más sofisticadas, así como los cuidados de enfermería específicos, como los encontrados en los trasplantes de órganos, la quimioterapia, cirugía cardiovascular, y otras terapias intensivas. Así, la enfermería tiene que preocuparse, en la actualidad, de monitorear el progreso y el cambio tecnológico, social y las crecientes demandas de la sociedad a fin de ofrecer una mejor atención. El mercado de trabajo para las enfermeras sigue las transformaciones de la globalización. Por lo tanto la inclusión de las enfermeras en el mundo del trabajo está directamente influida por el título profesional. Así que las competiciones están dirigidas a aquellos con una mejor preparación y conocimiento técnico y científico. 


\section{Enfermería en el proceso de especialización}

La Enfermería en respuesta al ritmo impuesto por el mundo del trabajo ha evolucionado, pasando por diversas etapas y transformaciones, siendo incorporada al nivel superior y más adelante al nivel de postgrado, con maestría y doctorado y otros tipos de especializaciones.

Los cursos de postgrado en Brasil están dentro de una concepción política de promoción del desarrollo económico en el país, por lo que se necesita una formación profesional de recursos humanos calificados, que pretende servir a dos demandas principales: "Las necesidades futuras de mano de obra calificada para cubrir los nuevos empleos creados por el desarrollo económico de planificación y la necesidad de científicos, investigadores y técnicos, capaces de desarrollar la investigación, esencial para el cambio. (10)

La formación de la enfermería se caracteriza por ser muy general, sin embargo, la existencia de cursos de postgrado permite a los profesionales especializarse en el área donde encuentran más afinidad con la carrera de Enfermería. Por lo tanto, se percibe que hay un desajuste entre la universidad idealizada y la realidad de la práctica, lo que ha supuesto una serie de dificultades para los nuevos graduados, la insatisfacción de empleadores y la falta de impacto de sus acciones sobre la salud. La desarmonía entre el aprendizaje académico y las expectativas en el campo de trabajo ha sido criticada en los círculos académicos de los graduados. (11)

Con la Ley de Directrices y Bases de Educación Nacional N $\cong 9394$ de $1996(\text { LDB })^{13}$, es evidente que las enfermeras a nivel de pregrado, deben prepararse adecuadamente, sea a través de cursos especializados, la mejora, ampliación, e incluso Masters y Ph.D, ya que el mercado laboral es cada vez más exigente. Aunque hay grupos interesados en la visión holística del hombre para la prestación de cuidados de enfermería, cada vez más las enfermeras están especializandose, como ocurre con otras profesiones de la salud.

Los programas de residencia en enfermería son buscados por la mayoría de las enfermeras como una excelente experiencia profesional. Especialmente la recién formada, se propone retardar la entrada en el mercado laboral en busca de mejores calificaciones. Sabemos que el tiempo para la realización de un solo curso de especialización, así como los factores relacionados con el fortalecimiento de la práctica, puede ser infinitamente menor que el tiempo y las oportunidades de una residencia.

\section{CONCLUSIÓN}

De este estudio se deducen con profundidad algunas de las características de la especialización en enfermería, porque la introducción de nuevas tecnologías ha producido y acelerado el proceso de transformación del mundo del trabajo, que busca cada vez más profesionales mejor preparados, refinados y especializados, ofreciendo la creación de muchas especialidades, incluida la enfermería.

La especialización ha contribuido eficazmente a la práctica de las enfermeras posibilitando su formación en diferentes áreas del conocimiento. Con respecto a la asistencia, sin duda, su contribución es incalculable, ya que permite a las personas ser asistidas con competencia. Por lo tanto, para la empresa, ofrece enfermeras 
interesadas, con experiencia y profesionales cualificados de acuerdo en las necesidades a la realidad y capaces de superar las dificultades prácticas de la salud entre la población con conocimiento y sensibilidad para mejorar la calidad de la enfermería en los servicios de salud.

Así, enfermeras y profesionales de la salud, buscan una nueva mejora, a través de cursos de especialización (postgrado lato sensu), ofrecidos actualmente. Una de las características encontradas se deriva de un panorama muy dinámico de las especialidades y las Sociedades de Expertos, que apunta a la reflexión y se refiere al mundo del trabajo, destinada a mejorar la integración técnica y diferenciada en el mercado laboral en un intento de hacerse valer en este mercado, que es cada vez más específico.

El perfil requerido por el mundo del trabajo obliga a las personas a tomar iniciativas y asumir responsabilidades, tener la capacidad de utilizar los instrumentos y equipos sofisticados e inteligentes, y familiarizarse con ellos, estar preparadas para el trabajo en equipo, puede llevar al liderazgo de este equipo y tener capacidad de planificar y ejecutar proyectos complejos. Por último tener la capacidad de aprender nuevas habilidades y adquirir actitudes de manera rápida y eficaz, que están abiertos a continuas transformaciones y diferentes formas de organización del trabajo y son capaces de identificar problemas y encontrar soluciones a estos problemas.

Por lo tanto, se concluye que en materia de salud, es necesario pensar en una formación capaz de ofrecer herramientas para el profesional para cuidar del ser humano, capaz de hacer frente a la vida y con todo todo lo que con ella está relacionado, de una manera ética y comprometida. Los datos indican la necesidad de repensar la formación de pregrado, no sólo con el objetivo de satisfacer las necesidades del mercado, sino sobre todo para moverse en busca de una formación capaz de actuar en esta realidad, especialmente cuando se trata de informar el futuro profesional con respecto a las posibilidades de formación continua.

\section{REFERÊNCIAS}

1. Paula MB, Santos VLCG. O significado de ser especialista para o enfermeiro estomaterapeuta. Rev. Latino-Am. Enfermagem. Vol. 11/2003, n 4, p. $474-482$. jil/ago. 2003. Disponível em: <http//www.scielo.br/scielo.php>. Acesso em: 27 março/ 2009.

2. Santos EF dos. A enfermagem no espelho: suas concepções sobre a profissão, a formação, a prática profissional e as especialidades. Tese (Doutorado em Enfermagem). Instituto de Medicina Social, Universidade Estadual do Rio de Janeiro, Rio de Janeiro: 2005.

3. Canatto FG de A. O residente de enfermagem e o mercado de trabalho: Expectativas e possibilidades concretas. Dissertação de Mestrado. Rio de Janeiro: Escola de Enfermagem Anna Nery/UFRJ, 1999.

4. Neves IG. As Especialidades e a atuação do enfermeiro no município do Rio de Janeiro. Trabalho de Conclusão de Curso - Graduação em Enfermagem Universidade Estácio de Sá. 2005. Orientadora: Geilsa Soraia C. Valente.

5. Minayo MCS. Pesquisa social: Teoria, Método e criatividade. Petrópolis: Vozes, 2007.

6. Markert M. O Desafio do conhecimento: Pesquisa qualitativa em saúde. 2 ed. Hucitec; São Paulo: 2003. 
7. Gutierrez MGR et al. Acompanhamento e avaliação da pós-graduação no Brasil: retrospectiva histórica da representação da enfermagem. Escola Anna Nery Revista de Enfermagem, Rio de Janeiro, Vol. 5 (2) Agosto 2001:161-62.

8. VIANA LO. A formação do enfermeiro no Brasil e as especialidades: 1920-1970. Rio de Janeiro: UFRJ/EEAN, 1995. Tese. (Doutorado em Enfermagem). Escola de Enfermagem Anna Nery, Universidade Federal do Rio de Janeiro, 1995. Orientadora: Maria Teresinha Pereira e Silva.

9. Oguisso T. Rumos da enfermagem: enfermeiras generalistas ou especialistas? Rev. Enfermagem UERJ, Rio de Janeiro, Vol. 9(2); may-ago; 2001:167-73.

10. Figueiredo NMA, Aguiar BGC. O impacto dos cursos de Especialização nos moldes de Residência em Enfermagem. Enf. Global. ㄲo 6 - May. 2005. Disponível em: www.um.es/eglobal. Acesso em 02/2010.

11. Erzinger AR, Trentini M. Enfermeiras e enfermeiros frente aos desafios no início da carreira profissional. Rev. Téc-cient.Enfermagem, Vol. 1(5); 2003: 332-39.

12. Ministério da Educação (Brasil). Lei de diretrizes e Bases da Educação no. 9.394/96. Disponível em: http://www.mec.gov.br. Acesso em 17-02-2010. 